\title{
Carbon Nanotubes for Potential Electronic and Optoelectronic Applications
}

\author{
Jia Chen \\ IBM T. J. Watson Research Center, 1101 Kitchawan Rd., Yorktown Heights, NY 10598 \\ Tel. 1 914.945.2046; Fax. 1914.945.4531; E-mail: chenjia@us.ibm.com
}

\begin{abstract}
Carbon nanotubes (CNTs) are novel quasi-one-dimensional materials with excellent electrical properties in addition to their remarkable mechanical strength, thermal conductivity and chemical inertness. Moreover, semiconducting CNTs are directgap semiconductors that directly absorb and emit light. This offers the possibility of developing a CNT-based electric and optoelectronic technology. [1]
\end{abstract}

\section{INTRODUCTION}

Significant progress has been made recently on carbon nanotube based field effect transistors (CNTFET), in terms of understanding both their scaling and performance limits [1]. However, the Schottky barriers (SB) formed between the CNT and the source/drain metal contacts lead to contacts-dominated switching, resulting in low drive current, undesirable subthreshold characteristics, and strong ambipolar conduction when the transistor is scaled down vertically [2], all unacceptable in logic applications. Here we report on chemical doping schemes utilizing molecules and a charge transfer mechanism to obtain self-aligned, stable and unipolar CNTFETs to meet the performance challenges. In the meanwhile, we can utilize both the ambipolar and unipolar conduction in CNTFETs for optoelectronic applications, and demonstrate spatially-resolved electron-hole recombination in long channel transistors, and impact-excited infrared emission in CNTs under high electric field. This allows us to efficiently generate high density of excitons in 1D and observe new phenomena that are not possible in bulk materials.

\section{CHARGE-TRANSFERRED DOPING}

Doing in CNTs is very different than that in silicon. The conventional ion-implantation method easily destroys CNT lattices. In this work, we utilize molecules to transfer charge to/from the surface of CNTs. We demonstrate an ability to change carrier injection properties; and to improve device performance in both ON- and OFF- transistor states. In particular, oxidizing and redox-active molecules are introduced to modify the workfunction of the source and drain electrodes of CNTFETs and, correspondingly, of their SBs. We have utilized triethyloxonium hexachloroantimonate (OA) $\left(\mathrm{C}_{2} \mathrm{H}_{5}\right)_{3} \mathrm{O}+\mathrm{SbCl}_{6}^{-}$to dope both the channel and the contacts of a CNTFET [3]. OA is a one-

Permission to make digital or hard copies of all or part of this work for personal or classroom use is granted without fee provided that copies are not made or distributed for profit or commercial advantage and that copies bear this notice and the full citation on the first page. To copy otherwise, to republish, to post on servers or to redistribute to lists, requires prior specific permission and/or a fee.

ICCAD'06, November 5-9, 2006, San Jose, CA

Copyright 2006 ACM 1-59593-389-1/06/0011...\$5.00 electron oxidant whose interaction with CNT leads to hole injection in the tube. Typical transfer characteristics before and after doping (Fig. 1a and 1b, respectively) shows the $V_{t h}$ for hole conduction in la creased from $-0.8 \mathrm{~V}$ to $0.05 \mathrm{~V}$; the $I_{o n}$, at constant overdrive, increased by about two orders of magnitude; the subthreshold swing $S=d V_{g} / d\left(\log I_{d}\right)$ decreased from $200 \mathrm{mV} / \mathrm{dec}$ to $85 \mathrm{mV} / \mathrm{dec}$; the minority carrier (electron current) injection at the drain successfully suppressed after doping and the transistor characteristics transformed from ambipolar to unipolar. Further study of selective doping of CNTFETs in the channel of the transistors and at the vicinity of the contacts indicated that doping in the channel contributed to increase of carrier density in the bulk of the tube, while doping at the contacts resulted in the modification of the work-function of the source and drain electrodes and, correspondingly, of their SBs. The modification of work-function involves the adsorption of the $\mathrm{SbCl}_{6}{ }^{-}$counter ions, which, with the induced image charge in the source/drain metal, produce an outward-directed dipole that increases local work-function. This increase favors hole injection at one electrode, while suppressing electron injection at the other. The important role of the SBs can be seen in Fig. 1 which shows the improvement of the $\mathbf{O N}$ - and OFF- state characteristics of a CNTFET upon doping with OA.
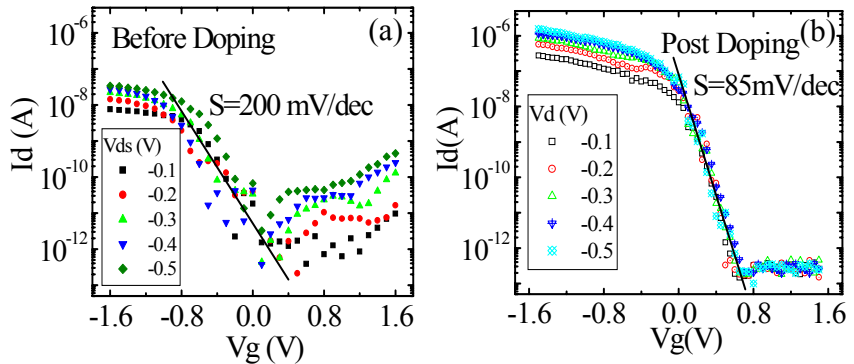

Figure 1 Transfer characteristics of a CNTFET before (a) and after (b) OA doping under various $V_{d s}$.

\section{SELF-ALIGNED CNTFETS}

When one increases the doping concentration, a p-CNTFET can be converted to an almost metallic tube. In this case, the SBs are ultimately minimized so the transport through the CNTFET is not limited by the contacts rather by the bulk of the tube, as in a conventional FET. Fig. 2 shows a top-gated device with selfaligned "extension-like" OA doping, realized by covering part of the channel of the CNT with oxide and an e-beam patterned tungsten (W) gate, leaving both the source/drain metals and the channel area close to the contacts un-gated and accessible for dopants (insets of Fig. 2). Before doping, the transistor was completely off due to the large SBs and lack of carriers in the un- 
gated regions; after doping, $\mathbf{O A}$ molecules modified the $\mathbf{S B s}$ at the contacts and increased carrier density on the un-gated section of the tube. The transistor was turned on as a p-FET with a $V_{\text {th }}$ of $0.2 \mathrm{~V}$, a sharp $S$ of $69 \mathrm{mV} / \mathrm{dec}$, and an excellent $I_{\text {on }} / I_{\text {off }}$ ratio of $10^{6}$. This is the first realization of a self-aligned top-gated CNTFET utilizing novel chemical doping [3].

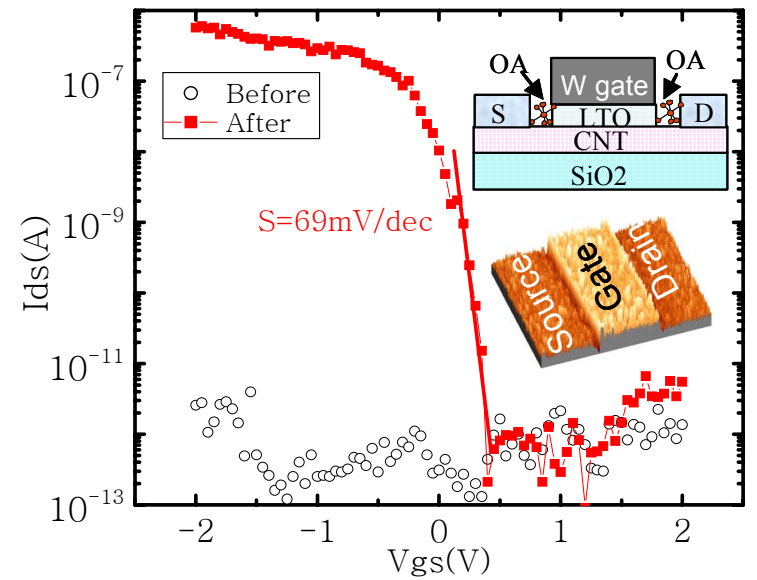

Figure 2 Transfer characteristics of a W-top-gated CNTFET before and after OA-doping at $V_{d s}=-0.5 \mathrm{~V} . S$ is $69 \mathrm{mV} /$ dec post doping. The inset shows a schematics and a SEM picture of the device.

To realize stable $\mathrm{n}$-doping of CNTs, we introduced aminecontaining hydrazine and observed similar device performance improvement in the doped CNTFETs [4]. We show in Fig. 3 the successful conversion of a p-CNTFET to n-type after doping, where we improved $I_{o n}$ by 3 orders of magnitude; suppressed the minority carrier (hole current) injection; and obtained the sharpest $S(87 \mathrm{mV} / \mathrm{dec})$ among n-doped devices.

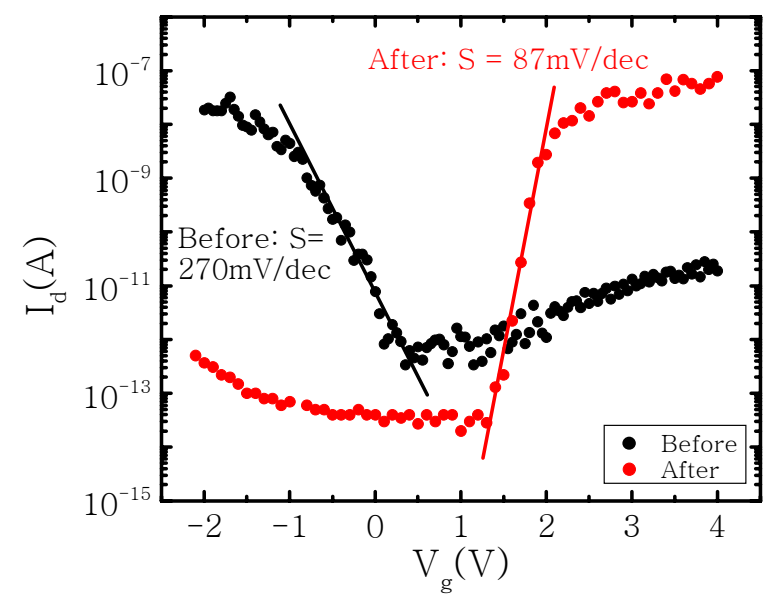

Figure 3 Transfer characteristics of a CNTFET before and after hydrazine-doping at $V_{d s}=0.5 \mathrm{~V} . S$ improved from the original 270 to $87 \mathrm{mV} / \mathrm{dec}$ after doping.

\section{ELECTRICALLY-INDUCED INFRARED EMISSION FROM CNTFETS}

Using ambipolar CNTFETs with appropriate biasing we can inject electrons and holes simultaneously from the source and drain electrodes of the nanotube [5]. CNTFETs provide a novel form of molecular light source that requires neither external doping nor well defined space charge region as in conventional LEDs. We find that most of the electroluminescence is confined to a small segment of the carbon nanotube that can be controllably positioned by varying the gate voltage, unique to $1 \mathrm{D}$ devices. The emission region can be moved over tens of microns in long CNTs by varying the gate potential [6].

In addition to electroluminescence in ambipolar CNTFETs, we also observed localized emission when biasing CNTFETs under high field. Fig. 4a shows an illustration of strongly enhanced infrared emission of a partially suspended CNTFET [7]. The different capacitive coupling between the Si back gate and the suspended and the supported part of the CNT leads to a local band-bending, which, in turn, generates a local high field under gate/drain biases. The carriers injected from the contacts can pick up enough energy under the local high field such that they can impact excite tightly-bound e-h pairs (excitons). Fig. $4 \mathrm{~b}$ shows that the locally generated infrared emission intensity increases exponentially with drive current. This is caused by the unique strong e-h binding (with an excitonic binding energy as large as $1 / 3$ of the energy band gap) and weak e-phonon scattering in 1D.
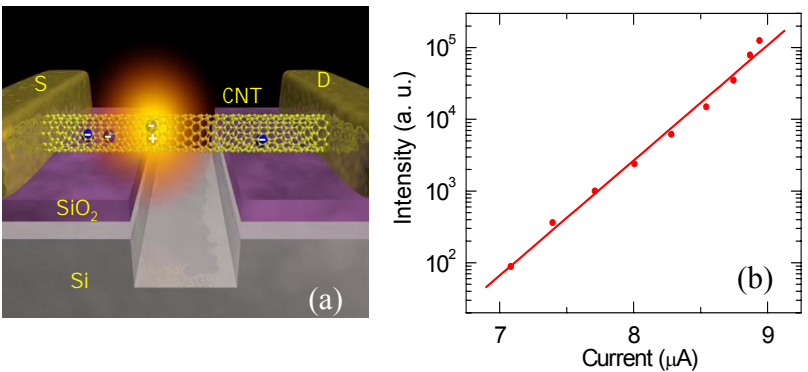

Figure 4 (a) Illustration depicting infrared emission from a partially suspended, back-gated CNTFET. (b) The impactexcited electroluminescence intensity (at $1.6 \mu \mathrm{m}$ ) increases exponentially with drive current at $V_{d}=-5 \mathrm{~V}$.

\section{ACKNOWLEDGMENTS}

The author thank Christian Klinke, Ali Afzali, Marcus Freitag on valuable inputs; Phaedon Avouris and Vasili Perebeinos for insightful discussions, and Qiang $\mathrm{Fu}$ and Prof. Jie Liu for providing the long CVD CNTs for the electroluminescence experiments.

\section{REFERENCES}

[1] Avouris, Ph. MRS Bull., 29, 2004, 403.

[2] Radosavljevic, M., et al., Appl. Phys. Lett., 83, 2003, 2435.

[3] Chen, J., et al., Appl. Phys. Lett., 86, 2005, 23108.

[4] Klinke, C., et al., Nano Lett., 5, 2005, 555.

[5] Misewich, J. A. et al., Science, 300, 2003, 783.

[6] Freitag, M., et. Al., Phys. Rev. Lett., 93, 2004, 0768031.

[7] Chen, J., et, al., Science, 301, 2005, 1171. 